PROCEEDINGS OF THE AMERICAN MATHEMATICAL SOCIETY

Volume 124, Number 10, October 1996

\title{
WEIGHTED COMPOSITION OPERATORS ON HILBERT SPACES OF VECTOR-VALUED FUNCTIONS
}

\author{
WILLIAM E. HORNOR AND JAMES E. JAMISON
}

(Communicated by Palle E. T. Jorgensen)

\begin{abstract}
In this paper we investigate criterion for the hyponormality, cohyponormality, and normality of weighted composition operators acting on Hilbert spaces of vector-valued functions.
\end{abstract}

\section{INTRODUCTION}

In recent years, considerable attention has been given to the delineation of weighted composition operators with regard to normality class. In short, given a weighted composition operator $W_{T} f=w f \circ T$ acting on the complex Hilbert space of square-integrable functions on a $\sigma$-finite measure space $(X, \Sigma, \mu)$, measure theoretic criteria have been found which determine the membership of $W_{T}$ in most of the major normality classes. These criteria involve objects $(\Sigma$-algebras and measurable functions) naturally associated with $w, T$, and $(X, \Sigma, \mu)$. In this note we consider the more general setting of weighted composition operators acting on $L^{2}\left(X, \Sigma, \mu, \mathbb{C}^{n}\right)$, the Hilbert space of $\Sigma$-measurable $\mathbb{C}^{n}$-valued functions $f$ for which $\|f(\cdot)\| \in L^{2}(X, \Sigma, \mu)$, where $\|\cdot\|$ represents the Euclidean norm. We obtain measure-theoretic criteria for the hyponormality of such operators and their adjoints.

Throughout this paper, $(X, \Sigma, \mu)$ is a complete, $\Sigma$-finite measure space, and $T$ is a nonsingular measurable transformation on $X$. We denote by $T^{-1} \Sigma$ the (completion of the) $\sigma$-subalgebra $\left\{T^{-1} A: A \in \Sigma\right\}$, and we assume that $h \doteq$ $\frac{d \mu \circ T^{-1}}{d \mu} \in L^{\infty}(X, \Sigma, \mu)$. For each $T^{-1} \Sigma$-measurable function $f$, there exists an essentially unique $\Sigma$-measurable function $g$ whose support lies in the support of $h$ with $f=g \circ T$. We write $g=f \circ T^{-1}$. See, for example, [3]. Of course this extends quite naturally to $T^{-1} \Sigma$-measurable matrix-valued functions. We denote by $\mathbf{L}^{2}$ the collection of (equivalence classes of) $\Sigma$-measurable $\mathbb{C}^{n}$-valued functions $f$ for which $\|f(\cdot)\|^{2}$ is $\mu$-integrable. Throughout the paper, $W$ will be an element of $\mathcal{M}_{n \times n} \doteq L^{\infty}\left(X, \Sigma, \mu, \mathbb{C}^{n \times n}\right)$, where $\mathbb{C}^{n \times n}$ is the space of $n \times n$ matrices with complex entries. We define a weighted composition operator $\mathbf{W}_{T}$ in $\mathbf{L}^{2}$ by

$$
\mathbf{W}_{T} f=W f \circ T \text {. }
$$

We remark that $W f \circ T$ denotes the product of the matrix-valued function $W$ with the vector-valued function $f \circ T$.

Received by the editors October 17, 1994 and, in revised form, February 21, 1995 and April 3, 1995.

1991 Mathematics Subject Classification. Primary 47B38, 47B20. 
In the study of weighted composition operators on $L^{2}(X, \Sigma, \mu, \mathbb{C})$, the notion of conditional expectation has proved indispensable. Using conditional expectations, it is possible to write $\left|W_{T}\right|$ as a multiplication operator on $L^{2}(X, \sigma, \mu, \mathbb{C})$ and to express $W_{T}^{*}$ in concrete form. These two applications of conditional expectation provide a starting point for the study of normality properties of weighted composition operators on $L^{2}(X, \sigma, \mu, \mathbb{C})$. We have a similar need for conditional expectations in our study of weighted composition operators on $\mathbf{L}^{2}$. Our extension of the concept of conditional expectation to matrix-valued functions is completely straightforward.

For each $f \in L^{p}(X, \Sigma, \mu), 1 \leq p \leq \infty$, there exists a unique $T^{-1} \Sigma$-measurable function $E(f)$ such that

$$
\int g f d \mu=\int g E(f) d \mu
$$

for every $T^{-1} \Sigma$-measurable function $g$ for which the left integral exists. The function $E(f)$ is called the conditional expectation of $f$ with respect to the subalgebra $T^{-1} \Sigma$. As an operator on $L^{p}(X, \Sigma, \mu), E$ is a contractive projection. The operator $E$ has the property that $E(k f)=k E(f)$ for all $T^{-1} \Sigma$-measurable functions $k$ for which $k f$ is integrable. For additional properties of the operator $E$, see [6] or [7].

Definition 1. Suppose $F$ is a $\Sigma$-measurable function taking on values in $\mathbb{C}^{m \times n}$, and that each component function $F_{i j}$ is in $L^{p_{i j}}(X, \Sigma, \mu, \mathbb{C}), 1 \leq p_{i, j} \leq \infty$. Then the conditional expectation of $F$ is the $T^{-1} \sum$-measurable $\mathbb{C}^{m \times n}$-valued function $\mathbf{E}_{m \times n}(F)$ whose $(i, j)$-th component function is $E\left(F_{i j}\right)$. That is, $\left(\mathbf{E}_{m \times n}(F)\right)_{i j}=$ $E\left(F_{i j}\right)$. In the following, we write $\mathbf{E}_{n}$ for $\mathbf{E}_{n \times 1}$.

It is a simple matter to compute $\left|\mathbf{W}_{T}\right|$ and $\mathbf{W}_{T}^{*}$ using conditional expectation and the change of variables formula. For any $f \in \mathbf{L}^{2}$, we have

$$
\begin{aligned}
\left\|\mathbf{W}_{T} f\right\|_{2}^{2} & =\int\langle W f \circ T, W f \circ T\rangle d u \\
& =\int\left\langle W^{*} W f \circ T, f \circ T\right\rangle d \mu \\
& =\int\left\langle\mathbf{E}_{n \times n}\left(W^{*} W\right) f \circ T, f \circ T\right\rangle d \mu \\
& =\int\left\langle h \mathbf{E}_{n \times n}\left(W^{*} W\right) \circ T^{-1} f, f\right\rangle d \mu .
\end{aligned}
$$

Setting $J \doteq h \mathbf{E}_{n \times n}\left(W^{*} W\right) \circ T^{-1}$, it follows that $\left|\mathbf{W}_{T}\right|=M_{J^{1 / 2}}$, where $M_{J^{1 / 2}}$ is the operator on $\mathbf{L}^{2}$ defined by

$$
M_{J^{1 / 2}} f=J^{1 / 2} f
$$

Also, for any $f, g \in \mathbf{L}^{2}$, we have

$$
\begin{aligned}
\left\langle\mathbf{W}_{T} f, g\right\rangle & =\int\langle W f \circ T, g\rangle d u=\int\left\langle f \circ T, W^{*} g\right\rangle d \mu \\
& =\int\left\langle f \circ T, \mathbf{E}_{n}\left(W^{*} g\right)\right\rangle d \mu=\int\left\langle f, h \mathbf{E}_{n}\left(W^{*} g\right) \circ T^{-1}\right\rangle d \mu,
\end{aligned}
$$

and it follows that

$$
\mathbf{W}_{T}^{*} g=h \mathbf{E}_{n}\left(W^{*} g\right) \circ T^{-1}
$$

for every $g \in \mathbf{L}^{2}$. 
Whenever $J$ occurs below, it will denote the function $h \mathbf{E}_{n \times n}\left(W^{*} W\right) \circ T^{-1}$. A classical result asserts that there exists an order preserving $*$-isomorphism between the $C^{*}$-algebra $L^{\infty}(X, \Sigma, d \mu)$ and the space $C(S)$ of continuous functions on a Stonian space $S$. (A convenient reference is [5].) By Corollary 3.3 of [4], $J$ admits a factorization

$$
J(x)=U^{*}(x) D(x) U(x)
$$

where $U(x)$ is unitary and $D(x)$ is diagonal for almost every $x \in X$. Let $\delta_{1}, \delta_{2}, \ldots, \delta_{n}$ denote the diagonal entries of $D$. We define a matrix-valued function $D^{\dagger}$ by

$$
D^{\dagger}(x)_{i j}= \begin{cases}\left(D(x)_{i j}\right)^{-1} & \text { if } D(x)_{i j} \neq 0 \\ 0 & \text { if } D(x)_{i j}=0\end{cases}
$$

and set $I_{D}=D^{\dagger} D$. For each natural number $k$, set $S_{k}=\left\{\left\|D^{\dagger}(x)\right\| \leq k\right\}$ and define $D_{k}^{\dagger}$ and $J_{k}^{\dagger}$ by $D_{k}^{\dagger}=\chi_{S_{k}} D^{\dagger}$ and $J_{k}^{\dagger}=U^{*} D_{k}^{\dagger} U$.

Next, we consider the product space $\bigoplus_{1}^{n} L^{2}\left(\delta_{i} d \mu\right) \doteq \bigoplus_{1}^{n} L^{2}\left(X, \Sigma, \delta_{i} d \mu\right)$ with the canonical Hilbert space norm $\|\cdot\|_{(n)}$. Define a mapping $\Lambda: \bigoplus_{1}^{n} L^{2}\left(\delta_{i} d \mu\right) \rightarrow$ $L^{0}\left(X, \Sigma, \mu, \mathbb{C}^{n}\right)$, the space of $\Sigma$-measurable $\mathbb{C}^{n}$-valued functions, by

$$
\Lambda g(x)=W(x)\left(U^{*} g\right) \circ T(x) .
$$

Choose an increasing sequence $\left\{X_{k}\right\}$ of measurable sets of finite measure with $\bigcup X_{k}=X$, and set $A_{k}=X_{k} \cap\{\|g \circ T\|<k\}$ and $g^{(k)}=\chi_{A_{k}} \cdot g$. Now

$$
\begin{aligned}
\|\Lambda\|_{2}^{2} & =\int\left\langle W\left(U^{*} g\right) \circ T, W\left(U^{*} g\right) \circ T\right\rangle d \mu \\
& =\int\left\langle U \circ T W^{*} W U^{*} \circ T g \circ T, g \circ T\right\rangle d \mu \\
& =\lim _{k \rightarrow \infty} \int\left\langle U \circ T W^{*} W U^{*} \circ T g^{(k)} \circ T, g^{(k)} \circ T\right\rangle d \mu \\
& =\lim _{k \rightarrow \infty} \int\left\langle U \circ T E_{n \times n}\left(W^{*} W\right) U^{*} \circ T g^{(k)} \circ T, g^{(k)} \circ T\right\rangle d \mu \\
& =\lim _{k \rightarrow \infty} \int\left\langle U h E_{n \times n}\left(W^{*} W\right) \circ T^{-1} U^{*} g^{(k)}, g^{(k)}\right\rangle d \mu \\
& =\int\left\langle h \mathbf{E}_{n \times n}\left(W^{*} W\right) \circ T^{-1} U^{*} g, U^{*} g\right\rangle d \mu \\
& =\int\left\langle U^{*} D U U^{*} g, U^{*} g\right\rangle d \mu=\int\langle D g, g\rangle d \mu=\|g\|_{(n)}^{2} .
\end{aligned}
$$

We see that $\Lambda$ is an isometry from $\bigoplus_{1}^{n} L^{2}\left(\delta_{i} d \mu\right)$ into $\mathbf{L}^{2}$.

Lemma 2. $\overline{\operatorname{ran}\left(\mathbf{W}_{T}\right)}=\operatorname{ran}(\Lambda)$.

Proof. Suppose $f \in \overline{\operatorname{ran}\left(\mathbf{W}_{T}\right)}$. Then there exists a sequence $\left\{f^{(k)}\right\}$ in $\mathbf{L}^{2}$ such that $W f^{(k)} \circ T \rightarrow f$ in norm. Now $\left\|U f^{(k)}\right\|_{2}=\left\|f^{(k)}\right\|_{2}$, and $\left\|U f^{(k)}-U f^{(j)}\right\|_{(n)}=$ $\left\|W f^{(k)} \circ T-W f^{(j)} \circ T\right\|_{2}$, so $\left\{U f^{(k)}\right\}$ is a Cauchy sequence in $\bigoplus_{1}^{n} L^{2}\left(\delta_{i} d \mu\right)$. Hence $\left\{U f^{(k)}\right\}$ converges in this space to some $g$, and by continuity, $f=\Lambda g$.

Next, suppose $g \in \bigoplus_{1}^{n} L^{2}\left(\delta_{i} d \mu\right)$. Then there exists a sequence $\left\{g^{(k)}\right\}$ in $\mathbf{L}^{2}$ with $g^{(k)} \rightarrow g$ in $\bigoplus_{1}^{n} L^{2}\left(\delta_{i} d \mu\right)$. Now $\left\{U^{*} g^{(k)}\right\}$ is also a sequence in $\mathbf{L}^{2}$, and by continuity $\Lambda g=\lim _{k \rightarrow \infty} W U^{*} \circ T g^{(k)} \circ T=\lim _{k \rightarrow \infty} W\left(U^{*} g^{(k)}\right) \circ T$, so $\Lambda g \in \overline{\operatorname{ran}\left(\mathbf{W}_{T}\right)}$. 


\section{A MEASURE-THEORETIC CHARACTERIZATION OF HYPONORMALITY}

In this section we present a measure-theoretic criterion for the hyponormality of $\mathbf{W}_{T}$. Our result is closely related to a theorem from [6].

Lemma 3. $\operatorname{ker} \mathbf{W}_{T}=U^{*}\left(\operatorname{ker} I_{D}\right)$.

Proof. We note that $f \in \operatorname{ker} \mathbf{W}_{T}$ if and only if

$$
\int\left\langle U^{*} D U f, f\right\rangle d \mu=\int\langle D U f, U f\rangle d \mu=0,
$$

that is, if and only if $U f \in \operatorname{ker} I_{D}$. This inclusion holds exactly when $f=U^{*} g$ for some $g \in \operatorname{ker} I_{D}$, and the result follows.

Lemma 4. $\overline{\operatorname{ran} \mathbf{W}_{T}^{*}}=U^{*}\left(\operatorname{ran} I_{D}\right)$, and $U^{*} I_{D} U$ is the projection onto $\overline{\operatorname{ran} \mathbf{W}_{T}^{*}}$.

Proof. Note that $k \in \overline{\operatorname{ran} \mathbf{W}_{T}^{*}}$ if and only if $k \in\left(\operatorname{ker} \mathbf{W}_{T}\right)^{\perp}$, and this is equivalent to

$$
\int\langle U k, g\rangle d \mu=\int\left\langle k, U^{*} g\right\rangle d \mu=0
$$

for every $g \in \operatorname{ker} I_{D}$. But this in turn is equivalent to $I_{D} U k=U k$, or $k=$ $U^{*} I_{D} U k$.

Lemma 5. The polar factorization of $\mathbf{W}_{T}$ is given by $\mathbf{W}_{T}=V M_{J^{1 / 2}}$, where

$$
V f=W\left(U^{*}\left(D^{\dagger}\right)^{1 / 2} U f\right) \circ T,
$$

and the adjoint of $V$ is given by

$$
V^{*} f=\left(U^{*}\left(D^{\dagger}\right)^{1 / 2} U\right) \mathbf{W}_{T}^{*} f .
$$

Proof. Let $V$ be as above. For any $f \in \mathbf{L}^{2}$, we have

$$
\begin{aligned}
\|V f\|^{2} & =\lim _{k \rightarrow \infty} \int_{T^{-1}\left(S_{k}\right)}\left\langle W\left(U^{*}\left(D^{\dagger}\right)^{1 / 2} U f\right) \circ T, W\left(U^{*}\left(D^{\dagger}\right)^{1 / 2} U f\right) \circ T\right\rangle d \mu \\
& =\lim _{k \rightarrow \infty} \int\left\langle W\left(U^{*}\left(D_{k}^{\dagger}\right)^{1 / 2} U f\right) \circ T, W\left(U^{*}\left(D_{k}^{\dagger}\right)^{1 / 2} U f\right) \circ T\right\rangle d \mu \\
& =\lim _{k \rightarrow \infty} \int\left\langle\mathbf{E}_{n \times n}\left(W^{*} W\right)\left(U^{*}\left(D_{k}^{\dagger}\right)^{1 / 2} U f\right) \circ T,\left(U^{*}\left(D_{k}^{\dagger}\right)^{1 / 2} U f\right) \circ T\right\rangle d \mu \\
& =\lim _{k \rightarrow \infty} \int\left\langle h \mathbf{E}_{n \times n}\left(W^{*} W\right) \circ T^{-1} U^{*}\left(D_{k}^{\dagger}\right)^{1 / 2} U f, U^{*}\left(D_{k}^{\dagger}\right)^{1 / 2} U f\right\rangle d \mu \\
& =\lim _{k \rightarrow \infty} \int_{S_{k}}\left\langle U^{*} I_{D} U f, f\right\rangle d \mu=\int\left\langle U^{*} I_{D} U f, f\right\rangle .
\end{aligned}
$$

It follows that $V$ is a partial isometry with initial space $\left(\operatorname{ker} \mathbf{W}_{T}\right)^{\perp}$ and final space $\overline{\operatorname{ran} \mathbf{W}_{T}}$. Employing Lemma 3, we see that $\mathbf{W}_{T}=V M_{J^{1 / 2}}$.

Denoting by $M$ the densely defined operator given by $M f=\left(U^{*}\left(D^{\dagger}\right)^{1 / 2} U\right) f$, we have $V=\mathbf{W}_{T} M$, and in light of the continuity of $\mathbf{W}_{T}$, we conclude that

$$
V^{*} f=\left(U^{*}\left(D^{\dagger}\right)^{1 / 2} U\right) \mathbf{W}_{T}^{*} f
$$

for every $f \in \mathbf{L}^{2}$.

Lemma 6. $\operatorname{ker} \mathbf{W}_{T} \subseteq \operatorname{ker} \mathbf{W}_{T}^{*}$ if and only if $W=U^{*} I_{D} U W$. 
Proof. We note that $\operatorname{ker} \mathbf{W}_{T} \subseteq \operatorname{ker} \mathbf{W}_{T}^{*}$ if and only if $\overline{\operatorname{ran} \mathbf{W}_{T}} \subseteq \overline{\operatorname{ran} \mathbf{W}_{T}^{*}}$, and this holds if and only if $W f \circ T=U^{*} I_{D} U W f \circ T$ for every $f \in \mathbf{L}^{2}$. Let $\xi \in \mathbb{C}^{n}$ and choose a sequence $\left\{A_{k}\right\}$ of $\Sigma$-measurable sets of finite measure with $\bigcup A_{k}=X$; we have $\chi_{T^{-1} A_{k}} W \xi=\chi_{T^{-1} A_{k}} U^{*} I_{D} U W \xi$ for each $k$. It follows that $W=U^{*} I_{D} U W$, and clearly if this last equality holds we must have $W f \circ T=U^{*} I_{D} U W f \circ T$ for every $f \in \mathbf{L}^{2}$.

Theorem 7. $\mathbf{W}_{T}$ is hyponormal if and only if the following two conditions hold:

(i) $W=U^{*} I_{D} U W$.

(ii) $J^{1 / 2} h \mathbf{E}_{n \times n}\left(W^{*} J_{k}^{\dagger} W\right) \circ T^{-1} J^{1 / 2} \leq J$ for all $k$.

Proof. Suppose (i) holds. Then

$$
\operatorname{ker} V^{*}=(\operatorname{ran} V)^{\perp}=\left(\operatorname{ran} \mathbf{W}_{T}\right)^{\perp}=\operatorname{ker} \mathbf{W}_{T}^{*} \supseteq \operatorname{ker} \mathbf{W}_{T}=U^{*}\left(\operatorname{ker} I_{D}\right),
$$

so the operator $U V^{*} U^{*}$ is well-defined on the subspace $\mathbf{L}^{2} \cap \bigoplus_{1}^{n} L^{2}\left(\delta_{i} d \mu\right)$ of $\bigoplus_{1}^{n} L^{2}\left(\delta_{i} d \mu\right)$. As $\mathbf{L}^{2}$ is dense in $\bigoplus_{1}^{n} L^{2}\left(\delta_{i} d \mu\right)$, we can regard $U V^{*} U^{*}$ as a densely defined operator in $\bigoplus_{1}^{n} L^{2}\left(\delta_{i} d \mu\right)$. We note that for $f \in \mathbf{L}^{2},\left\|U V^{*} U^{*} f\right\|_{(n)}=$ $\left\|\mathbf{W}_{T}^{*} U^{*} f\right\|_{2}$ and $\|f\|_{(n)}=\left\|\mathbf{W}_{T} U^{*} f\right\|_{2}$, and consequently that $U V^{*} U^{*}$ is a contraction in $\bigoplus_{1}^{n} L^{2}\left(\delta_{i} d \mu\right)$ if and only if $\mathbf{W}_{T}$ is hyponormal. If $U V^{*} U^{*}$ extends to a continuous operator on $\bigoplus_{1}^{n} L^{2}\left(\delta_{i} d \mu\right)$, then for any $f \in \mathbf{L}^{2}$ and $g \in \bigoplus_{1}^{n} L^{2}\left(\delta_{i} d \mu\right)$, we have

$$
\begin{aligned}
\left\langle U V^{*} U^{*} f, g\right\rangle_{(n)} & =\int\left\langle D\left(D^{\dagger}\right)^{1 / 2} U \mathbf{W}_{T}^{*} U^{*} f, g\right\rangle d \mu \\
& =\int\left\langle D f, D^{\dagger} U W_{T} U^{*} D^{1 / 2} g\right\rangle d \mu .
\end{aligned}
$$

It will follow that the adjoint of $U V^{*} U^{*}$ in $\bigoplus_{1}^{n} L^{2}\left(\delta_{i} d \mu\right)$ is given by $D^{\dagger} U \mathbf{W}_{T} U^{*} D^{1 / 2}$ provided $D^{\dagger} U \mathbf{W}_{T} U^{*} D^{1 / 2}$ maps $\bigoplus_{1}^{n} L^{2}\left(\delta_{i} d \mu\right)$ into itself. For any $f \in \bigoplus_{1}^{n} L^{2}\left(\delta_{i} d \mu\right)$, we have

$$
\begin{aligned}
\| D^{\dagger} U & \mathbf{W}_{T} U^{*} D^{1 / 2} f \|_{(n)}^{2}=\lim _{k \rightarrow \infty} \int\left\langle D_{k}^{\dagger} U \mathbf{W}_{T} U^{*} D^{1 / 2} f, U \mathbf{W}_{T} U^{*} D^{1 / 2} f\right\rangle d \mu \\
= & \lim _{k \rightarrow \infty} \int\left\langle D_{k}^{\dagger}(W)\left(U^{*} D^{1 / 2} f\right) \circ T,(U W)\left(U^{*} D^{1 / 2} f\right) \circ T\right\rangle d \mu \\
= & \lim _{k \rightarrow \infty} \int\left\langle(U W)^{*} D_{k}^{\dagger}(U W)\left(U^{*} D^{1 / 2} f\right) \circ T,\left(U^{*} D^{1 / 2} f\right) \circ T\right\rangle d \mu \\
= & \lim _{k \rightarrow \infty} \int\left\langle\mathbf{E}_{n \times n}\left((U W)^{*} D_{k}^{\dagger}(U W)\right)\left(U^{*} D^{1 / 2} f\right) \circ T,\left(U^{*} D^{1 / 2} f\right) \circ T\right\rangle d \mu \\
= & \lim _{k \rightarrow \infty} \int\left\langle h \mathbf{E}_{n \times n}\left((U W)^{*} D_{k}^{\dagger}(U W)\right) \circ T^{-1}\left(U^{*} D^{1 / 2} f\right),\left(U^{*} D^{1 / 2} f\right)\right\rangle d \mu \\
& =\lim _{k \rightarrow \infty} \int\left\langle D^{1 / 2} U h \mathbf{E}_{n \times n}\left(W^{*} J_{k}^{\dagger} W\right) \circ T^{-1} U^{*} D^{1 / 2} f, f\right\rangle d \mu .
\end{aligned}
$$

We observe that $D^{\dagger} U \mathbf{W}_{T} U^{*} D^{1 / 2}$ is a contraction on $\bigoplus_{1}^{n} L^{2}\left(\delta_{i} d \mu\right)$ if and only if

$$
D^{1 / 2} U h \mathbf{E}_{n \times n}\left(W^{*} J_{k}^{\dagger} W\right) \circ T^{-1} U^{*} D^{1 / 2} \leq D
$$

holds for all $k$, and this is equivalent to (ii) above.

We note that the continuity of $U \mathbf{W}_{T} U^{*}$ in $\bigoplus_{1}^{n} L^{2}\left(\delta_{i} d \mu\right)$ is equivalent to the existence of a constant $c$ such that $\left\|\mathbf{W}_{T}^{*} f\right\|_{2} \leq c\left\|\mathbf{W}_{T} f\right\|_{2}$ for all $f \in \mathbf{L}^{2}$. This in turn implies that the family $\left\{D^{1 / 2} U h \mathbf{E}_{n \times n}\left(W^{*} J_{k}^{\dagger} W\right) \circ T^{-1} U^{*} D^{1 / 2}\right\}_{k=1}^{\infty}$ is an increasing 
sequence of operators on $\mathbf{L}^{2}$ bounded above by the operation of multiplication by $c D$. It follows that $\left\{D^{1 / 2} U h \mathbf{E}_{n \times n}\left(W^{*} J_{k}^{\dagger} W\right) \circ T^{-1} U^{*} D^{1 / 2}\right\}_{k=1}^{\infty}$ converges in the strong operator topology on $\mathcal{B}\left(\mathbf{L}^{2}\right)$. As the space of operators consisting of multiplication by $n \times n$ matrices of $L^{\infty}$ functions is a von Neumann algebra, we see that $\left|D^{\dagger} U \mathbf{W}_{T} U^{*} D^{1 / 2}\right|$ is given by a matrix multiplication on $\bigoplus_{1}^{n} L^{2}\left(\delta_{i} d \mu\right)$.

Example 8. We take as our measure space $(X, \Sigma, \mu)$ the set of real numbers with Lebesgue measure. Our transformation $T: \mathbb{R} \rightarrow \mathbb{R}$ is given by $T(x)=x+1$, so that $T^{-1} \Sigma=\Sigma$ and $\mathbf{E}_{2 \times 2}$ is the identity operator. Suppose $\varphi_{1}$ and $\varphi_{2}$ are positive, bounded, and decreasing functions on $\mathbb{R}$, define $W \in \mathcal{M}_{2 \times 2}$ by

$$
W=\left(\begin{array}{ll}
\sqrt{\phi_{1} \circ T}+\sqrt{\phi_{2} \circ T} & \sqrt{\phi_{1} \circ T}-\sqrt{\phi_{2} \circ T} \\
\sqrt{\phi_{1} \circ T}-\sqrt{\phi_{2} \circ T} & \sqrt{\phi_{1} \circ T}+\sqrt{\phi_{2} \circ T}
\end{array}\right),
$$

and let $\mathbf{W}_{T}: L^{2}\left(X, \Sigma, \mu, \mathbb{C}^{2}\right) \rightarrow L^{2}\left(X, \Sigma, \mu, \mathbb{C}^{2}\right)$ be defined in the usual way. It is easy to see that $J=U^{*} D U$ where

$$
U=U^{*}=\frac{1}{\sqrt{2}}\left(\begin{array}{cc}
1 & 1 \\
1 & -1
\end{array}\right), \quad D=\left(\begin{array}{cc}
\varphi_{1} & 0 \\
0 & \varphi_{2}
\end{array}\right),
$$

and that $I_{D}$ is the $2 \times 2$ identity matrix. It can be shown that (i) and (ii) of the above theorem become

(i) $T^{-1}\left(\operatorname{supp}\left(\varphi_{i}\right)\right) \subseteq \operatorname{supp}\left(\varphi_{i}\right)$.

(ii) $^{\prime} \varphi_{i}(x) / \varphi_{i}(x-1)<1$.

Since each $\varphi_{i}$ is a decreasing function with full support, both of these two conditions are satisfied.

The above theorem is a generalization of a theorem of Lambert [6] for weighted composition operators on $L^{2} \doteq L^{2}(X, \Sigma, \mu)$. The simpler formulation of the theorem in the scalar setting stems primarily from the fact that nonnegative functions are conditionable, and hence the family of inequalities in Theorem 7 can be replaced with a single inequality. Also, in the scalar setting we have no need for diagonalization methods, and our operator $I_{D}$ is simply multiplication by the characteristic function of $\operatorname{supp}(J)$. We state his theorem as a corollary.

Corollary 9. The operator $W_{T} \in \mathcal{B}\left(L^{2}\right)$ is hyponormal if and only if the following two conditions hold:

(i) $\operatorname{supp}(w) \subset \operatorname{supp}(J)$.

(ii) $h E\left(\frac{|w|^{2}}{J}\right) \circ T^{-1} \leq \chi_{\operatorname{supp}(J)}$.

\section{Characterizations of COHYPONORMal AND NORMAL OPERATORS}

In this section we develop necessary and sufficient conditions for the cohyponormality of $\mathbf{W}_{T}$. (Recall that $\mathbf{W}_{T}$ is cohyponormal if $\mathbf{W}_{T}^{*}$ is hyponormal.) Our result is related to a theorem from [1]. In the following, $\Sigma_{0} \doteq\{A \in \Sigma: \mu(A)<\infty\}$.

Lemma 10. $\operatorname{ker} \mathbf{W}_{T}^{*} \subseteq \operatorname{ker} \mathbf{W}_{T}$ if and only if for every $A \in \Sigma_{0}$ there exists a $\Sigma$-measurable matrix-valued function $G_{A}$ such that

$$
U^{*} I_{D} U \cdot \chi_{A}=W\left(U^{*} G_{A}\right) \circ T
$$

where each column $\left[G_{A}\right]_{k}$ of $G_{A}$ is an element of $\bigoplus_{1}^{n} L^{2}\left(\delta_{i} d \mu\right)$.

Proof. We note that the above set inclusion is equivalent to $\operatorname{ran} \mathbf{W}_{T}^{*} \subseteq \overline{\operatorname{ran} \mathbf{W}_{T}}$, and this holds if and only if for every $A \in \Sigma_{0}$ and $\xi \in \mathbb{C}^{n}$ there exists a $g_{A, \xi} \in$ $\bigoplus_{1}^{n} L^{2}\left(\delta_{i} d \mu\right)$ with $U^{*} I_{D} U \cdot \chi_{A} \xi=W\left(U^{*} g_{A, \xi}\right) \circ T$. This holds if and only if for each 
$A \in \Sigma_{0}$ and $k \in\{1,2, \ldots, n\}$ there exists $g_{A, \xi}^{(k)} \in \bigoplus_{1}^{n} L^{2}\left(\delta_{i} d \mu\right)$ with $\chi_{A}\left[U^{*} I_{D} U\right]_{k}=$ $W\left(U^{*} g_{A, \xi}^{(i)}\right) \circ T$. But this is equivalent to the existence of the matrix $G_{A}$ above.

Theorem 11. $\mathbf{W}_{T}$ is cohyponormal if and only if the following two conditions hold:

(i) For every $A \in \Sigma_{0}$ there exists a $\Sigma$-measurable matrix-valued function $G_{A}$ such that $\left[G_{A}\right]_{k} \in \bigoplus_{1}^{n} L^{2}\left(\delta_{i} d \mu\right)$ for each $k$ and $U^{*} I_{D} U \cdot \chi_{A}=W\left(U^{*} G_{A}\right) \circ T$.

(ii) $J^{2} \geq h \mathbf{E}_{n \times n}\left(W^{*} J W\right) \circ T^{-1}$.

Proof. By the above lemma, (i) is equivalent to the inclusion $\operatorname{ker} \mathbf{W}_{T}^{*} \subseteq \operatorname{ker} \mathbf{W}_{T}$, a condition clearly necessary for the cohyponormality of $\mathbf{W}_{T}$. Now if (i) holds, it follows that $\mathbf{W}_{T}$ is cohyponormal if and only if $\left\|\mathbf{W}_{T}^{*} \mathbf{W}_{T} f\right\|_{2} \geq\left\|\mathbf{W}_{T} \mathbf{W}_{T} f\right\|_{2}$ for every $f \in \mathbf{L}^{2}$. We note that

$$
\left\|\mathbf{W}_{T}^{*} \mathbf{W}_{T} f\right\|_{2}^{2}=\|J f\|_{2}^{2}=\int\left\langle J^{2} f, f\right\rangle d \mu
$$

while

$$
\left\|\mathbf{W}_{T} \mathbf{W}_{T} f\right\|_{2}^{2}=\int\langle J W f \circ T, W f \circ T\rangle d \mu=\int\left\langle h \mathbf{E}_{n \times n}\left(W^{*} J W\right) \circ T^{-1} f, f\right\rangle d \mu .
$$

So if (i) above holds, then $\mathbf{W}_{T}$ is cohyponormal if and only if (ii) holds.

A scalar version of Theorem 11 is given in Theorem 4.2 of [1]. Condition (i) above reduces to a condition concerning $\sigma$-algebras and the second condition is replaced by a simple functional inequality. If $\mathcal{A} \subset \Sigma$ is a $\sigma$-algebra and $C \subset X$, then $\mathcal{A}_{C} \doteq\{A \cap C: A \in \mathcal{A}\}$.

Corollary 12. $W_{T}^{*}$ is hyponormal if and only if the following two conditions hold:

(i) $\Sigma_{\operatorname{supp}(J)} \subset\left(T^{-1} \Sigma\right)_{\operatorname{supp}(w)}$.

(ii) $J \leq J \circ T$.

Theorem 13. $\mathbf{W}_{T}$ is normal if and only if

(i) For every $A \in \Sigma_{0}$ there exists a $\Sigma$-measurable matrix-valued function $G_{A}$ such that $\left[G_{A}\right]_{k} \in \bigoplus_{1}^{n} L^{2}\left(\delta_{i} d \mu\right)$ for each $k$ and $U^{*} I_{D} U \cdot \chi_{A}=W\left(U^{*} G_{A}\right) \circ T$.

(ii) $J^{2}=h \mathbf{E}_{n \times n}\left(W^{*} J W\right) \circ T^{-1}$.

Proof. Again, (i) is equivalent to the inclusion $\operatorname{ker} \mathbf{W}_{T}^{*} \subseteq \operatorname{ker} \mathbf{W}_{T}$. Under this condition, the normality of $\mathbf{W}_{T}$ is equivalent to the equality $\left\|\mathbf{W}_{T}^{*} \mathbf{W}_{T} f\right\|_{2}=\left\|\mathbf{W}_{T} \mathbf{W}_{T} f\right\|_{2}$ for all $f \in \mathbf{L}^{2}$. The short calculation involved in the previous theorem shows this to be equivalent to (ii).

Example 14. We take as our measure space the interval $[0,1]$ with Lebesgue measure. Our transformation $T$ is defined by $T(x)=1-x$, so $T^{-1} \Sigma=\Sigma$ and $\mathbf{E}_{2 \times 2}$ is the identity operator. We define $W \in \mathcal{M}_{2 \times 2}$ by

$$
W=\left(\begin{array}{cc}
0 & \cos x \\
\cos (1-x) & 0
\end{array}\right)
$$

and define $\mathbf{W}_{T}$ in the usual fashion. Now

$$
J=\left(\begin{array}{cc}
\cos ^{2} x & 0 \\
0 & \cos ^{2}(1-x)
\end{array}\right),
$$


and $I_{D}$ is the $2 \times 2$ identity matrix. Condition (i) above is satisfied for any Lebesgue measurable set $A$ by taking $G_{A}=\left(\chi_{A} W^{-1}\right) \circ T$. A straightforward calculation yields

$$
J^{2}=\left(\begin{array}{cc}
\cos ^{4} x & 0 \\
0 & \cos ^{4}(1-x)
\end{array}\right)=h \mathbf{E}_{2 \times 2}\left(W^{*} J W\right) \circ T^{-1} .
$$

Hence the operator $\mathbf{W}_{T}$ is normal, this in spite of the fact that the weight function fails to be normal.

The scalar analogue of Theorem 13 is considerably simpler and avoids the use of conditional expectations. The characterization of normal weighted composition operators on $L^{2}(X, \Sigma, \mu)$ is given by Theorem 2.14 of [2], which we state below.

Theorem 15. Suppose $\operatorname{supp}(w) \subset T^{-1}(\operatorname{supp}(w))$. Then $W_{T}$ is normal if and only if the following two conditions hold:

(i) $\Sigma_{\operatorname{supp}(w)} \subset\left(T^{-1} \sigma\right)_{\operatorname{supp}(w)}$.

(ii) $\chi_{\operatorname{supp}(w)} h w^{2} \circ T^{-1}=h \circ T w^{2}$.

If $\operatorname{supp}(w) \subset T^{-1}(\operatorname{supp}(w))$ fails to hold, then $W_{T}$ cannot be normal.

\section{REFERENCES}

[1] J. Campbell and W. Hornor, Seminormal composition operators, J. Operator Theory 29 (1993), 323-343. MR 95f:47052

[2] J. Campbell and W. Hornor, Localising and seminormal composition operators on $L^{2}$, Proc. Roy. Soc. Edinburgh 124A (1994), 301-316. MR 95d:47033

[3] J. Campbell and J. Jamison, On some classes of weighted composition operators, Glasgow Math. J. 32 (1990), 82-94. MR 91m:47040

[4] D. Deckard and C. Pearcy, On matrices over the ring of continuous complex valued functions on a Stonian space, Proc. Amer. Math. Soc. 14 (1963), 322-328. MR 26:5438

[5] R. Kadison and J. Ringrose, Fundamentals of the Theory of Operator Algebras, Vol. I, Academic Press, New York, 1983. MR 85j:46099

[6] A. Lambert, Hyponormal composition operators, Bull. London Math. Soc. 18 (1986), 395-400. MR 87h:47059

[7] M. M. Rao, Measure Theory and Integration, Wiley-Interscience, New York, 1987. MR 89k:28001

Department of Mathematics, University of Southern Mississippi, Hattiesburg, MisSISSIPPI 39406

Department of Mathematical Sciences, University of Memphis, Memphis, Tennessee 38152 